PART I

STELLAR FLUXES 
A. ABSOLUTE CALIBRATION 


\title{
ABSOLUTE INTENSITY CALIBRATION METHODS IN THE VACUUM UV REGION
}

\author{
G. BOLDT ${ }^{\dagger}$ \\ Max-Planck-Institut für Physik und Astrophysik, \\ Institut für extraterrestrische Physik, \\ 8046 Garching b. München, W. Germany
}

\begin{abstract}
As a summary of the principal results presented at the ESRO symposium on Calibration Methods in the Vacuum Ultra Violet (Munich, 1968) a description is given of three different absolute intensity calibration methods. These are the branching ratio method, the synchrotron radiation method and the black-body radiation method, and they define the present state of the art.
\end{abstract}

\section{Introduction}

Since space technology opened the possibility of observing stars and stellar systems in the ultraviolet, the question of intensity calibration in that region has become of special interest to astronomers. As a result of this interest, the members of the ESRO Working Group on 'Stars and Stellar Systems' under the chairmanship of Prof. Gratton agreed at the end of 1967 to organize a symposium on calibration methods in the UV in order to find out the present state of the art. This meeting was held in Munich in May 1968 and gave a good idea about what is going on in this field at different laboratories [1].

When considering intensity calibration problems one has to discuss two questions, namely how to produce an intensity standard and how to calibrate an astronomical instrument with the help of the intensity standard. The first of these two points is the more fundamental problem and in accordance with its aim, the symposium was more or less related to this point.

An intensity standard may be realized by a source or by a detector, but in any case it has to fulfil the following conditions:

(1) All parameters which influence the intensity of the source, or as the case may be the efficiency of the detector, must be known.

(2) The dependence of the intensity or of the efficiency on these parameters has to be known.

(3) It must be possible to adjust and to check all these parameters in a simple way, at any time, with tolerances that guarantee an acceptable precision in intensity or efficiency.

Starting from these conditions the study of all so-called intensity standards leads to the result that development based on sources should be preferred to one based on detectors. Therefore the following description of some intensity standards takes only sources into consideration.

+ Deceased January 18, 1970.

Houziaux and Butler (eds.), Ultraviolet Stellar Spectra and Ground-Based Observations, 5-11. All Rights Reserved. Copyright (C) 1970 by the IAU. 


\section{Production of Intensity Standards}

The present state of the art in the field of intensity standards is defined by three different methods which are all based on sources. These are:

(1) The branching ratio method (the first description of the method was given by Griffin and McWhirter from the Culham group).

(2) The synchrotron radiation method (here the work which has been done at DESY in Hamburg in cooperation with the Heidelberg group by Haensel, Labs, Lembke and Pitz has especially to be mentioned).

(3) The black-body radiation method (which has been developed by Boldt in Munich).

\section{A. THE BRANCHING RATIO METHOD}

Figure 1 illustrates the principle of this method and, as an example of its application, the calibration of a UV monochromator carried out by McWhirter and co-workers in Culham.

In principle one needs two spectral lines coming from the same upper level. One of these lines must lie in the visible or near UV of the spectrum, where its intensity can be measured by comparison with an approved intensity standard (tungsten ribbon lamp or carbon arc). The other line must lie in the vacuum UV. If the ratio between the transition probabilities of these two lines is known and if, furthermore, the plasma emitting these lines is optically thin for both lines so that proportionality between intensity and transition probability is known, then the intensity of the UV line can be calculated.

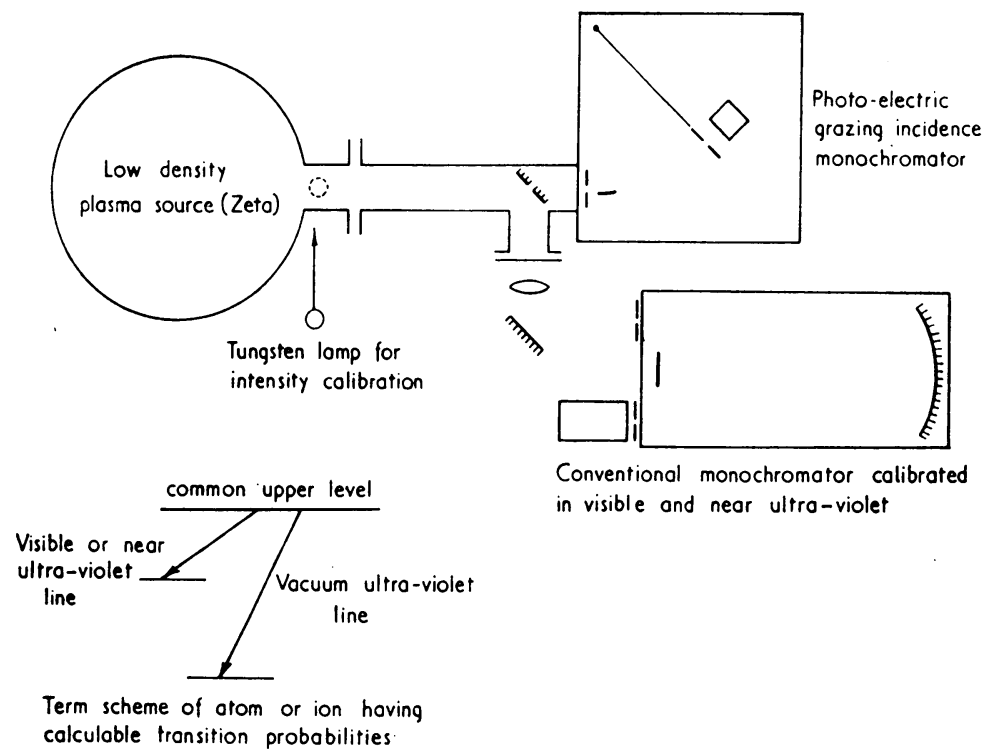

Fig. 1. The principle of the branching ratio method and an example of its application. 
The instrumental equipment for the application of this method uses ZETA as the source, with a grazing incidence monochromator for the vacuum UV and a conventional monochromator for the visible and near UV. Light coming from the plasma is split into two beams by a mirror system, so that the UV line and the visible line can be measured simultaneously. After this the plasma source is replaced by a tungsten ribbon lamp which calibrates the monochromator for the visible light.

\section{TABLE I}

List of lines used in the branching ratios calibration

\begin{tabular}{|c|c|c|c|}
\hline Transition & $\lambda(\AA)$ & $w$ (upper) & $A\left(\sec ^{-1}\right)$ \\
\hline $1-3$ & 1025.72 & 18 & $5.575 \times 10^{7}$ \\
\hline $2-3$ & 6562.80 & 18 & $4.410 \times 10^{7}$ \\
\hline HeII $1-4$ & 243.03 & 32 & $1.278 \times 10^{7 \mathrm{a}}$ \\
\hline $2-4$ & 1215.1 & 32 & $8.419 \times 10^{6 \mathrm{a}}$ \\
\hline $3-4$ & 4685.68 & 32 & $8.986 \times 10^{6 a}$ \\
\hline HeII 1-5 & 237.33 & 50 & $4.125 \times 10^{6 \mathrm{a}}$ \\
\hline $3-5$ & 3203.15 & 50 & $2.201 \times 10^{6 \mathrm{a}}$ \\
\hline He II $1-3$ & 256.32 & 18 & $5.575 \times 10^{7 \mathrm{a}}$ \\
\hline $2-3$ & 1640.4 & 18 & $4.410 \times 10^{7 \mathrm{a}}$ \\
\hline CiV $2 s-3 p$ & 312.43 & 6 & $4.56 \times 10^{9}$ \\
\hline $3 s-3 p$ & 5801.51 & 4 & $3.19 \times 10^{7}$ \\
\hline$N \vee 2 s-3 p$ & 209.28 & 6 & $1.20 \times 10^{10}$ \\
\hline $3 s-3 p\left(P_{3 / 2}\right)$ & 4603.83 & 4 & $4.15 \times 10^{7}$ \\
\hline$O v I 2 s-3 p$ & 150.10 & 6 & $2.59 \times 10^{10}$ \\
\hline $3 s-3 p\left(P_{3 / 2}\right)$ & 3811.35 & 4 & $5.10 \times 10^{7}$ \\
\hline
\end{tabular}

Table I gives a list of lines which have been used for this calibration method by McWhirter and co-workers.

It is obvious that ZETA can be replaced by other sources, e.g. by a hollow cathode lamp (this has already been done by McWhirter and other experimenters). Furthermore, we can expect that the list of lines will be extended.

In any case the branching ratio method is an elegant one for transfering the approved methods of absolute intensity calibration in the visible to the vacuum UV region.

The error of this method is mainly given by the uncertainty in our knowledge of transition probability ratios and by the uncertainty in the visible intensity standard. The impression is that further development of this method should finally lead to an accuracy of $\pm 10 \%$ to $\pm 20 \%$.

\section{B. THE SYNCHROTRON RADIATION METHOD}

Our knowledge about the synchrotron radiation is based on the Schwinger theory, which describes the radiation being emitted by a circulating relativistic electron. A number of investigations have shown that this theory can be applied to synchrotron radiation with very good accuracy. Consequently the relative intensity distribution 
mainly depends on the radius of the synchrotron and on the end energy of the electron and can therefore be calculated.

Figure 2 shows the result of such calculations applied to the DESY machine and taking into consideration special observational conditions. As one can see, there is only a very weak dependence on the end energy of the electron. This is also true for shorter wavelengths outside this diagram. The reason for this is the very high energy of the synchrotron, by which the maximum intensity is shifted to very short wavelengths. The maxima of the curves shown in the diagram lie between 0.3 and $30 \AA$.

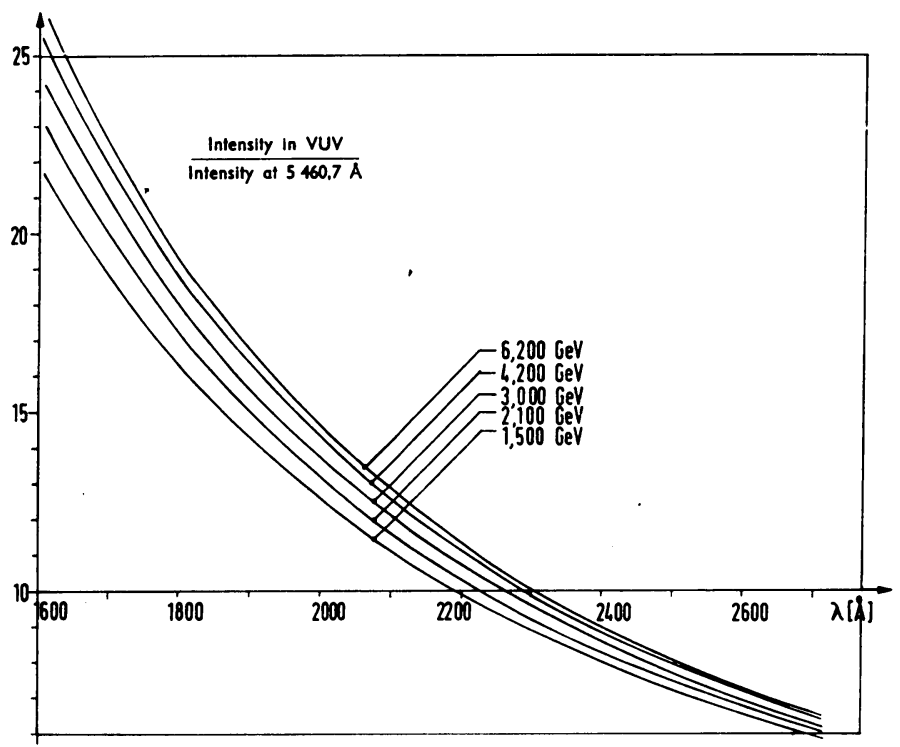

Fig. 2. Relative intensity distribution of the DESY synchrotron radiation (calculated for special observational conditions).

Hence exact knowledge of the energy is not critical if one restricts the observation of the synchrotron radiation to the vacuum UV. Consequently the synchrotron is a very excellent source for relative intensity calibrations in the range between the visible and vacuum UV.

In order to get absolute intensity values of the synchrotron radiation one has to measure the number of electrons in the synchrotron, which is proportional to the absolute intensity of the radiation. The accuracy of such measurements is not better than \pm 20 to $\pm 25 \%$. This means that the error in the absolute intensity of the synchrotron radiation will also be at least \pm 20 to $\pm 25 \%$.

Better results can be obtained by absolute calibration of the synchrotron radiation in the visible with the help of an approved intensity standard source (e.g. tungsten ribbon lamp). Because of its very good quality as a relative intensity standard, the synchrotron can then transfer the absolute calibration from the visible to the vacuum UV. This procedure has been carried out at DESY. 
The accuracy which can be achieved in this way for absolute vacuum UV intensities can be estimated to be about $\pm 10 \%$.

\section{THE BLACK-BODY RADIATION METHOD}

The source which can produce black-body radiation is a wall-stabilized cascade arc such as is shown in Figure 3 (see reference for further details). The plasma in the arc-channel having a pressure of $1 \mathrm{~atm}$ is thermal and consists mainly of nitrogen and carbon. These two elements emit a certain number of optically thick lines in the

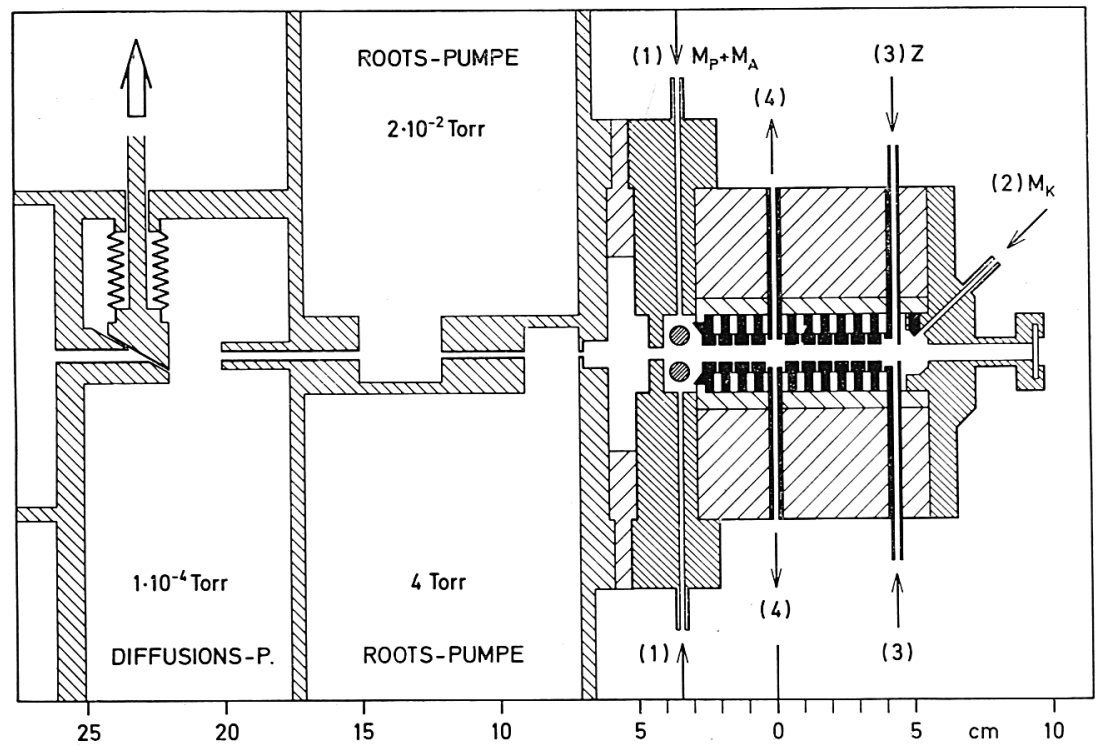

Fig. 3. Wall-stabilized cascade arc for producing black-body radiation in the far UV.

region from 1100 to $2500 \AA$. These lines can leave the plasma without disturbance by absorption and self-absorption via an argon window, which is realized by an argon plasma flow from the end of the arc-channel to the gas outlet in the middle part of the channel.

Since the absolute intensity in the centre of the optically thick lines is given by the Planck function, one has only to measure the plasma temperature in order to get the absolute intensities. This has been done using four different spectroscopic methods. The result was $12540 \mathrm{~K}$ with an accuracy of \pm 1 to $\pm 2 \%$. Consequently the black body intensities are known with an accuracy of \pm 10 to $\pm 20 \%$.

The arc plasma is connected to the vacuum system by a three stage differential pumping system.

Figure 4 shows the Planck function for $12540 \mathrm{~K}$, which is the temperature of the arc plasma. On this curve the positions of the observed optically thick lines are indicated. The interpolation between these points provides the absolute intensity scale 


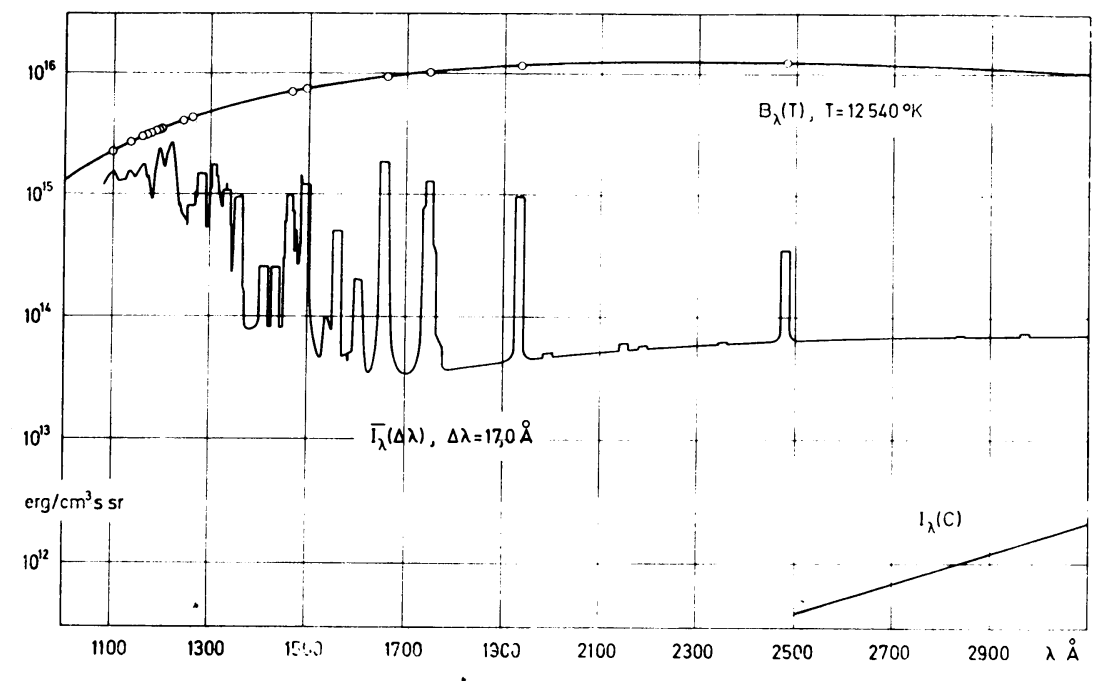

Fig. 4. Planck function of $12540 \mathrm{~K}$, absolute intensity distribution in the arc spectrum and absolute intensity distribution in the spectrum of the carbon arc.

at any point of the spectrum between 1100 and $2500 \AA$. This enables us to evaluate the absolute intensity distribution of the whole arc spectrum between 1100 and $2500 \AA$. The curve denoted by $I_{\lambda}(\Delta \lambda)$ shows the result of this operation, where a spectral resolution of $17.0 \AA$ has been chosen. It stands to reason that this can be done also for other resolutions.

Attention should be drawn to the continuum between 1700 and $3100 \AA$. The intensity of this continuum has been measured between 1700 and $2500 \AA$, put into a potential law, and then extrapolated up to $3100 \AA$. Since no remarkable jump of the continuum intensity is to be expected in this region, this extrapolation is acceptable.

Thus one has finally obtained the absolute intensity distribution in the whole arc spectrum between 1100 and $3100 \AA$ without using any other intensity standard.

The curve in the lower part of Figure 4, denoted by $I_{\lambda}(C)$, gives the absolute intensity distribution of the carbon arc, which is a well-known intensity standard in the visible and near UV. The comparison of the radiation of both sources in the overlapping region between 2500 and $3100 \AA$ is consistent within limits of $1 \%$ at $2500 \AA$ and $5 \%$ at $3100 \AA$. From this result one may gather that the estimated accuracy of the absolute intensity in the arc spectrum $( \pm 10$ to $\pm 20 \%)$ is realistic or perhaps even a little too pessimistic.

\section{Conclusion}

The fundamental problem in the field of absolute intensity calibration is the development of primary standards. The present state of the art in this field is defined by three different methods, which are all based on sources and which should be able to guarantee an accuracy of the order of $\pm 10 \%$. 
The next aim should be to compare these methods with each other in order to find out if they really agree within the estimated error limits. In the present situation these comparison experiments are of much more importance than the development of other intensity standards.

\section{Reference}

[1] 'Calibration Methods in the Ultraviolet and X-Ray Regions of the Spectrum', ESRO SP-33, December 1968.

\section{Discussion}

Malaise: In order to use the monochromatic flux as a calibrated source, you should be able to put the two curves ( $B_{\lambda}$ and actual output) of the figure on the same intensity scale. To do so, you have to measure the efficiency of the spectrograph at least for one wavelength, can you indicate how this was done?

Boldt: All curves shown in the diagram are related to the source-intensity-output. They do not give any information on the efficiency of the monochromator. 